\title{
Isolation, Structural Elucidation, and $\alpha$-Glucosidase Inhibitory Activities of Triterpenoid Lactones and Their Relevant Biogenetic Constituents from Ganoderma resinaceum
}

\author{
Xian-Qiang Chen ${ }^{1}$, Li-Gen Lin ${ }^{1}{ }^{10}$, Jing Zhao ${ }^{1,2, *}$, Ling-Xiao Chen ${ }^{1}$, Yu-Ping Tang ${ }^{3}$, \\ De-Lun Luo ${ }^{2}$ and Shao-Ping $\mathrm{Li}^{1}{ }^{1} *$ \\ 1 State Key Laboratory of Quality Research in Chinese Medicine, Institute of Chinese Medical Sciences, \\ University of Macau, Macau 999078, China; yb27528@umac.mo (X.-Q.C.); LigenL@umac.mo (L.-G.L.); \\ yb47517@umac.mo (L.-X.C.) \\ 2 Innovative Institute of Chinese Medicine and Pharmacy, Chengdu University of Traditional Chinese \\ Medicine, Chengdu 611730, China; ldl@mirbay.com \\ 3 Jiangsu Collaborative Innovation Center of Chinese Medicinal Resources Industrialization, Jiangsu Key \\ Laboratory for High Technology Research of TCM Formulae, National and Local Collaborative Engineering \\ Center of Chinese Medicinal Resources Industrialization and Formulae Innovative Medicine, Nanjing \\ University of Chinese Medicine, Nanjing 210023, China; yupingtang@njutcm.edu.cn \\ * Correspondence: zhaojing.cpu@163.com or jingzhao@umac.mo (J.Z.); \\ spli@umac.mo or spli@hotmail.com (S.-P.L.); Tel.: +853-8822-4692 (S.-P.L.); Fax: +853-2884-1358 (S.-P.L.)
}

Received: 15 May 2018; Accepted: 5 June 2018; Published: 8 June 2018

\begin{abstract}
Ganoderma resinaceum has been used as an ethnomedicine for lowering blood sugar. To clarify the bioactive chemical constituents contributing to lower blood sugar, chemical investigation on the fruiting bodies of Ganoderma resinaceum was conducted by chromatographic techniques, and led to the isolation of 14 compounds. Their structures were elucidated as triterpenoid lactones (1-4 and 8 ) and ganoderma acids (5-7 and 9-14) based on the analysis of extensive spectroscopy (mass spectrometry (MS), nuclear magnetic resonance (NMR), infrared (IR), and ultraviolet (UV)) and comparison with literature data. Compounds 3, 5, 6, and 9-14 were evaluated for $\alpha$-glucosidase inhibitory activity. Compounds 1-7 are new compounds. Compounds $\mathbf{1 - 4}$ and $\mathbf{8}$ were characteristic of an oxaspirolactone moiety, consisting of a five-membered ether ring, a five-membered lactone ring, and a characteristic C-23 spiro carbon. It is rare for natural products that such an oxaspirolactone moiety occurred in the lanostane-type triterpenoids. Compounds 5-7 and 9-14 may be important intermediates of the biosynthetic pathways of 1-4 and 8. Compounds 1 and $\mathbf{2}$ showed more potent inhibitory activity against $\alpha$-glucosidase compared with the positive control drug acarbose with $\mathrm{IC}_{50}$ value of $0.75 \pm 0.018 \mathrm{mM}$ and $1.64 \pm 0.022 \mathrm{mM}$, respectively.
\end{abstract}

Keywords: Ganoderma resinaceum; triterpenoid; triterpenoid lactone; oxaspirolactone; $\alpha$-glucosidase inhibitory activity

\section{Introduction}

Ganoderma resinaceum has long been used as an ethnomedicine to treat hyperglycemia, liver diseases, and immunoregulation [1,2]. Lanostane-type triterpenoids [2,3], nortriterpenoids [4], meroterpenoids [5], steroids [6], phenolic compounds [7], and polysaccharides [8] had been reported from the fruiting bodies and cultured mycelia of G. resinaceum. These metabolites and crude extracts of G. resinaceum showed various potential biological activities, such as anticancer $[9,10]$, 
hepatoprotection [2], antimicrobial activity [11], antioxidant activity [7], and inhibitory activities against acetyl cholinesterase, tyrosinase, $\alpha$-amylase, and $\alpha$-glucosidase [3,7].

Our team has reported 48 triterpenoids, 16 nortriterpenoids, 9 meroterpenoids, and 8 steroids isolated from the fruiting bodies of G. resinaceum in the previous studies [3-6]. As a continuous research to investigate biological secondary metabolites of G. resinaceum, phytochemical investigation of $G$. resinaceum led to the isolation and identification of seven new triterpenoids (1-7) and seven known compounds, 8-14 (Figure 1). Compounds 1-4 and 8, triterpenoid lactones, contain an oxaspirolactone moiety consisting of a five-membered ether ring, a five-membered lactone ring, and a characteristic C-23 spiro carbon, which is rare for lanostane-type triterpenoids from natural products. Herein, we report the isolation and structural elucidation of triterpenoid lactones (1-4 and 8) and their relevant biogenetic chemical constituents (5-7 and 9-14), and their $\alpha$-glucosidase inhibitory activities.
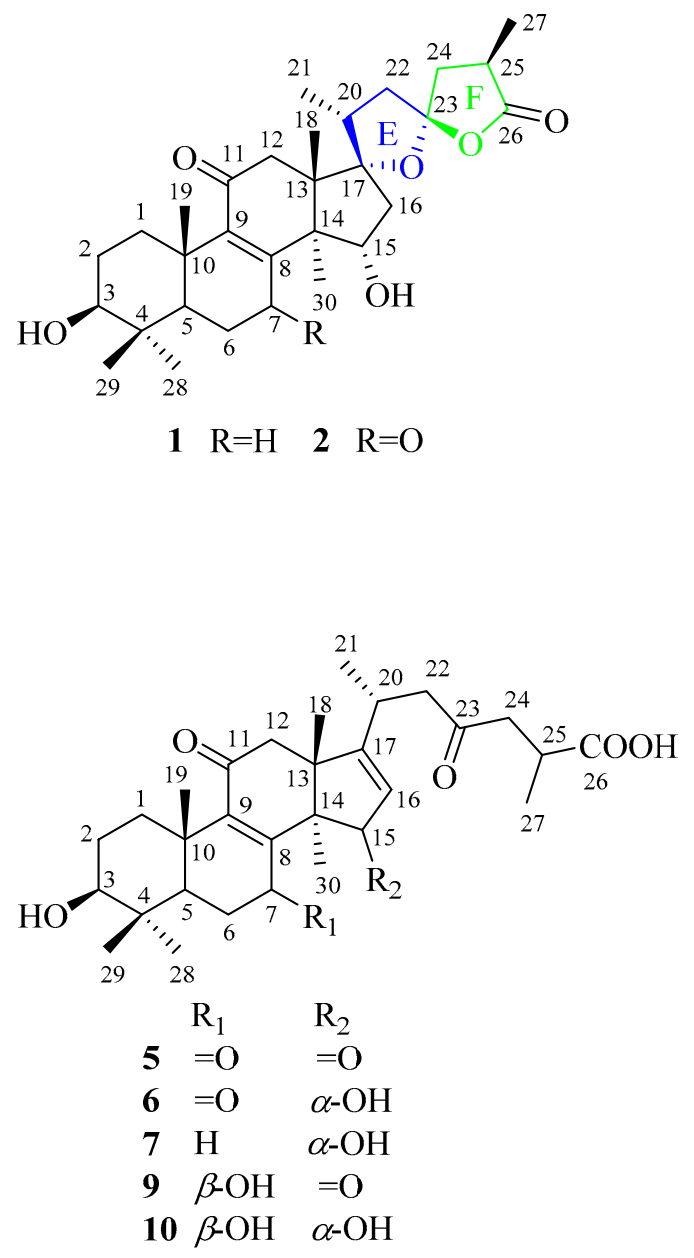
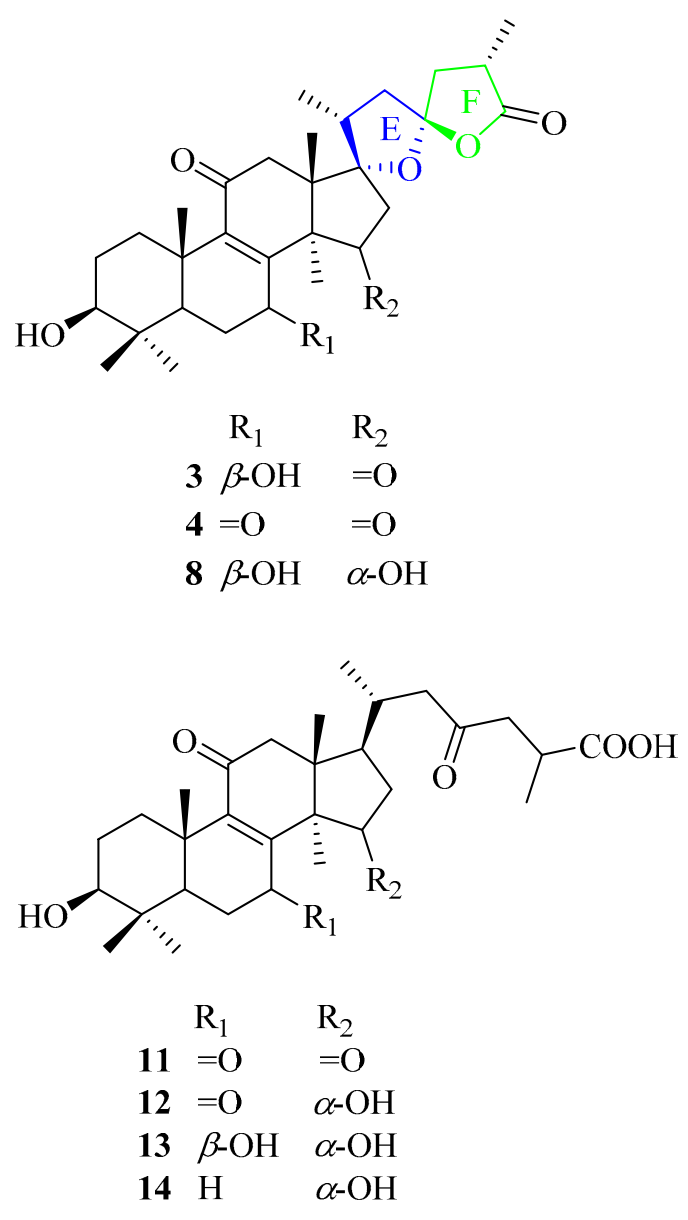

Figure 1. Structure of compounds 1-14.

\section{Results}

Fourteen compounds were isolated from the ethanol extract of G. resinaceum by various column chromatography, including silica gel, ODS gel, MCI gel, Sephadex LH-20, and semipreparative high performance liquid chromatography (HPLC). Their structures were identified as triterpenoid lactones (1-4 and 8) and ganoderma acids (5-7 and 9-14). Compounds 1 and 2 are strong $\alpha$-glucosidase inhibitors. Structural elucidation of new compounds (1-7) and bioassay are as follows. 


\subsection{Structural Elucidation}

Compound 1 was obtained as a white powder. Its molecular formula was defined as $\mathrm{C}_{30} \mathrm{H}_{44} \mathrm{O}_{6}$ by high resolution electrospray ionization mass spectrometry (HRESIMS) at $m / z 499.3033$ [M-H] ${ }^{-}$ (calcd for $\mathrm{C}_{30} \mathrm{H}_{43} \mathrm{O}_{6}, 499.3065$ ) and 1D NMR data (Table 1). Its IR spectrum showed the presence of hydroxy (3471 $\left.\mathrm{cm}^{-1}\right)$, carbonyl $\left(1767 \mathrm{~cm}^{-1}\right)$, and $\alpha, \beta$-unsaturated carbonyl $\left(1635 \mathrm{~cm}^{-1}\right)$ groups. The UV spectrum showed an absorption band to $258 \mathrm{~nm}$ due to the presence of $\alpha, \beta$-unsaturated carbonyl group. The ${ }^{1} \mathrm{H}$ NMR spectrum displayed seven methyl signals $\left[\delta_{\mathrm{H}} 1.15(3 \mathrm{H}, \mathrm{s}), 1.42(3 \mathrm{H}, \mathrm{s}), 0.91(3 \mathrm{H}, \mathrm{d})\right.$, $1.23(3 \mathrm{H}, \mathrm{d}), 1.27(3 \mathrm{H}, \mathrm{s}), 1.13(3 \mathrm{H}, \mathrm{s})$, and $1.68(3 \mathrm{H}, \mathrm{s})]$ and two oxygenated methine signals $\left(\delta_{\mathrm{H}} 3.52\right.$ and 4.72). The ${ }^{13} \mathrm{C}$ NMR and HSQC spectra revealed the presence of 30 carbon signals consisting of seven methyls, eight methylenes, five methines including two oxygenated methines at $\delta_{\mathrm{C}} 77.8$ and 72.9 , six $\mathrm{sp}^{3}$ quaternary carbons (two oxygenated carbon signals at $\delta_{\mathrm{C}} 95.6$ and 113.3), one ketone group $\left(\delta_{C} 198.6\right)$, one carboxylic group $\left(\delta_{C} 178.7\right)$, and two olefinic carbons $\left(\delta_{C} 165.2\right.$ and 139.9$)$. The above spectroscopic data suggested 1 to be lanostane-type triterpenoid. The rings A-D were established by the heteronuclear multiple bond correlation (HMBC) and ${ }^{1} \mathrm{H}-{ }^{1} \mathrm{H}$ correlation spectroscopy (COSY) experiment (Figure 2). The key HMBC correlations from H-28 $\left(\delta_{\mathrm{H}} 1.27\right)$ and $\mathrm{H}-29\left(\delta_{\mathrm{H}} 1.13\right)$ to $\mathrm{C}-3\left(\delta_{\mathrm{C}}\right.$ 77.8), combined with ${ }^{1} \mathrm{H}-{ }^{1} \mathrm{H}$ COSY correlations of $\mathrm{H}-3\left(\delta_{\mathrm{H}} 3.52\right)$ with $\mathrm{H}-2\left(\delta_{\mathrm{H}} 1.99\right.$ and 2.06), confirmed the presence of $3-\mathrm{OH}$. The $\alpha, \beta$-unsaturated ketone system at C-8/C-9/C-11 was assigned by the HMBC correlations from H-7 $\left(\delta_{\mathrm{H}} 2.96\right.$ and 2.76$)$ to $\mathrm{C}-8\left(\delta_{\mathrm{C}} 165.2\right)$ and $\mathrm{C}-9\left(\delta_{\mathrm{C}} 139.9\right), \mathrm{H}-19\left(\delta_{\mathrm{H}} 1.42\right)$ and H-12 $\left(\delta_{\mathrm{H}} 2.46\right)$ to $\mathrm{C}-9\left(\delta_{\mathrm{C}} 139.9\right)$, and $\mathrm{H}-12\left(\delta_{\mathrm{H}} 3.38\right.$ and 2.46$)$ to $\mathrm{C}-11\left(\delta_{\mathrm{C}} 198.6\right)$. The 15-OH was established on the basis of the HMBC correlations of $\mathrm{H}-15\left(\delta_{\mathrm{H}} 4.72\right)$ with C-8 $\left(\delta_{\mathrm{C}} 165.2\right), \mathrm{C}-14\left(\delta_{\mathrm{C}} 54.4\right), \mathrm{C}-16\left(\delta_{\mathrm{C}} 47.3\right)$, and C-30 $\left(\delta_{\mathrm{C}} 21.8\right)$, and $\mathrm{H}-30\left(\delta_{\mathrm{H}} 1.68\right)$ with C-15 $\left(\delta_{\mathrm{C}} 72.9\right)$, as well as the COSY cross peaks of $\mathrm{H}-15\left(\delta_{\mathrm{H}}\right.$ 4.72) with $\mathrm{H}-16\left(\delta_{\mathrm{H}} 2.52\right.$ and 2.82). Apart from seven degrees of unsaturation ascribing to the rings A-D, two carbonyls, and one double bond, the remaining two degrees of unsaturation indicated that 1 should have two rings in the side chain. According to a carboxylic carbon $\left(\delta_{\mathrm{C}} 178.7\right)$, an acetal secondary carbon $\left(\delta_{\mathrm{C}} 113.3\right)$, and a downfield oxygenated $\mathrm{sp}^{3}$ tertiary carbon $\left(\delta_{\mathrm{C}} 95.6\right)$, it was assumed that the structure of 1 possessed a novel oxaspirolactone moiety consisting of a five-membered ether ring, a five-membered lactone ring, and a characteristic C-23 spiro carbon. The hypothesis of oxaspirolactone moiety was supported by the HMBC correlations from H-21 $\left(\delta_{\mathrm{H}} 0.91\right)$ to C-17 $\left(\delta_{\mathrm{C}} 95.6\right), \mathrm{C}-20\left(\delta_{\mathrm{C}} 43.7\right)$, and C-22 $\left(\delta_{\mathrm{C}} 44.62\right), \mathrm{H}-27\left(\delta_{\mathrm{H}} 1.23\right)$ to $\mathrm{C}-24\left(\delta_{\mathrm{C}} 44.61\right), \mathrm{C}-25\left(\delta_{\mathrm{C}} 35.7\right)$, and C-26 $\left(\delta_{\mathrm{C}} 178.7\right), \mathrm{H}-22\left(\delta_{\mathrm{H}} 2.64\right.$ and 1.76) and $\mathrm{H}-24\left(\delta_{\mathrm{H}} 2.36\right.$ and 1.96) to C-23 $\left(\delta_{\mathrm{C}} 113.3\right)$, together with the COSY correlations of H-21 $\left(\delta_{\mathrm{H}} 0.91\right)$ and $\mathrm{H}-22\left(\delta_{\mathrm{H}} 2.64\right)$ with $\mathrm{H}-20\left(\delta_{\mathrm{H}} 2.14\right)$, and $\mathrm{H}-24\left(\delta_{\mathrm{H}} 2.36\right.$ and 1.96$)$ and $\mathrm{H}-27\left(\delta_{\mathrm{H}} 1.23\right)$ with $\mathrm{H}-25\left(\delta_{\mathrm{H}} 3.02\right)$. The planar structure of 1 was highly similar to that of ganotropic acid (8), except for the disappearance of hydroxy group attached to C-7 in $\mathbf{1}$.

The absolute configuration of C-17 and C-23 has an important bearing on chemical shifts of C-20, $\mathrm{C}-22$, and C-24 due to the anisotropic effect of the lactone oxygen atom. The ${ }^{13} \mathrm{C}$ NMR chemical shifts of C-20, C-22, and C-24 for $(17 S, 23 S)$-isomer were almost equal, however, those of $(17 S, 23 R),(17 R, 23 S)$, and $(17 R, 23 R)$-isomers showed dramatical differences larger than 5 ppm [12,13]. Therefore, the absolute configurations of C-17 and C-23 for 1 were highly close to that of $(17 S, 23 S)$-isomer reported in the literature [12], and both determined to be $S$-configuration based on comparison of NMR spectroscopic data $\left(\mathrm{C}-20\left(\delta_{\mathrm{C}} 43.4\right), \mathrm{C}-22\left(\delta_{\mathrm{C}} 44.6\right)\right.$, and C-24 $\left.\left(\delta_{\mathrm{C}} 44.9\right)\right)$ with those of similar structures. The Me-21 and Me-27 were assigned as $\alpha$ - and $\beta$-orientation, respectively, based on the ROESY correlations of $\mathrm{H}-18\left(\delta_{\mathrm{H}} 1.15\right)$ with $\mathrm{H}-20\left(\delta_{\mathrm{H}} 2.14\right), \mathrm{H}-24 \alpha\left(\delta_{\mathrm{H}} 2.36\right)$ with $\mathrm{H}-21\left(\delta_{\mathrm{H}} 0.91\right)$ and $\mathrm{H}-25\left(\delta_{\mathrm{H}} 3.02\right)$, and $\mathrm{H}-24 \beta$ $\left(\delta_{\mathrm{H}} 1.96\right)$ with $\mathrm{H}-27\left(\delta_{\mathrm{H}} 1.23\right)$. The key ROESY correlations of H-19 $\left(\delta_{\mathrm{H}} 1.42\right)$ with $\mathrm{H}-29\left(\delta_{\mathrm{H}} 1.13\right)$, H-3 $\left(\delta_{\mathrm{H}} 3.52\right)$ with H-5 $\left(\delta_{\mathrm{H}} 1.18\right)$ and H-28 $\left(\delta_{\mathrm{H}} 1.27\right)$, and $\mathrm{H}-18\left(\delta_{\mathrm{H}} 1.15\right)$ with $\mathrm{H}-15\left(\delta_{\mathrm{H}} 4.72\right)$ assigned $3-\mathrm{OH}$ and $15-\mathrm{OH}$ as $\beta$ - and $\alpha$-orientation, respectively. Accordingly, compound 1 was identified as (17S,23S)-17,23-epoxy-3 $\beta, 15 \alpha$-dihydroxy-11-oxo-5 $\alpha$-lanosta-8-en-26,23-olide.

Compound 2 was isolated as a white powder. Its molecular formula was determined to be $\mathrm{C}_{30} \mathrm{H}_{42} \mathrm{O}_{7}$ due to the HRESIMS ion peak at $m / z 513.2852[\mathrm{M}-\mathrm{H}]^{-}$(calcd for $\mathrm{C}_{30} \mathrm{H}_{41} \mathrm{O}_{7}, 513.2852$ ), implying ten degrees of unsaturation. The IR and UV spectra showed the presence of hydroxy $\left(3442 \mathrm{~cm}^{-1}\right)$, carbonyl $\left(1767 \mathrm{~cm}^{-1}\right)$, and $\alpha, \beta$-unsaturated carbonyl $\left(1665 \mathrm{~cm}^{-1}\right.$ and $\left.272 \mathrm{~nm}\right)$ groups. 
The ${ }^{1} \mathrm{H}$ NMR, ${ }^{13} \mathrm{C}$ NMR (Table 1), and HSQC spectra indicated the presence of seven methyls $\left(\delta_{\mathrm{H}} 0.89(3 \mathrm{H}, \mathrm{s}), 1.01(3 \mathrm{H}, \mathrm{d}), 1.03(3 \mathrm{H}, \mathrm{s}), 1.05(3 \mathrm{H}, \mathrm{s}), 1.24(3 \mathrm{H}, \mathrm{s}), 1.25(3 \mathrm{H}, \mathrm{d})\right.$, and $1.29(3 \mathrm{H}$, $\mathrm{s})$ ), seven methylenes, five methines including two oxygenated methine at $\delta_{\mathrm{C}} 77.3$ and 72.7 , six $\mathrm{sp}^{3}$ quaternary carbons including two oxygenated quaternary carbons at $\delta_{\mathrm{C}} 94.7$ and 112.7, one tetrasubstituted olefinic group $\left(\delta_{\mathrm{C}} 150.3\right.$ and 154.5), and three carbonyl groups $\left(\delta_{\mathrm{C}} 205.0,201.8\right.$, and 178.5). The abovementioned data suggested 2 to be similar to 1 except for the presence of an additional ketone in 2. The HMBC correlations from $\mathrm{H}-5\left(\delta_{\mathrm{H}} 1.55\right)$ and $\mathrm{H}-6\left(\delta_{\mathrm{H}} 2.56\right.$ and 2.57$)$ to $\mathrm{C}-7\left(\delta_{\mathrm{C}} 205.0\right)$ confirmed that a ketone was located at C-7. The relative configuration of 2 was established based on the rotating-frame nuclear overhauser effect correlation spectroscopy (ROESY) experiment, coupling constant, and comparison of its NMR spectroscopic data with that of $\mathbf{1}$. The $3-\mathrm{OH}$ was determined to be $\beta$-oriented by the ROESY correlations (Figure 2$)$ and the larger coupling constant $\left(\delta_{\mathrm{H}} 3.29\right.$, dd, $J=11.4,4.8 \mathrm{~Hz})$. The ROESY correlation of $\mathrm{H}-18\left(\delta_{\mathrm{H}} 1.05\right)$ with $\mathrm{H}-15\left(\delta_{\mathrm{H}} 4.45\right)$ assigned $15-\mathrm{OH}$ as $\alpha$-orientation. the Me-21 and Me-27 was established as $\alpha$-orientation and $\beta$-orientation, respectively, based on the ROESY correlations of H-20 $\left(\delta_{\mathrm{H}} 2.26\right)$ with $\mathrm{H}-18\left(\delta_{\mathrm{H}} 1.05\right), \mathrm{H}-21\left(\delta_{\mathrm{H}} 1.01\right)$ with $\mathrm{H}-24 \alpha$ $\left(\delta_{\mathrm{H}} 2.47\right)$, and $\mathrm{H}-24 \beta\left(\delta_{\mathrm{H}} 2.03\right)$ with $\mathrm{H}-27\left(\delta_{\mathrm{H}} 1.25\right)$. Therefore, compound 2 was determined to be (17S,23S)-17,23-epoxy-3 $\beta, 15 \alpha$-dihydroxy-7,11-dioxo-5 $\alpha$-lanosta-8-en-26,23-olide.

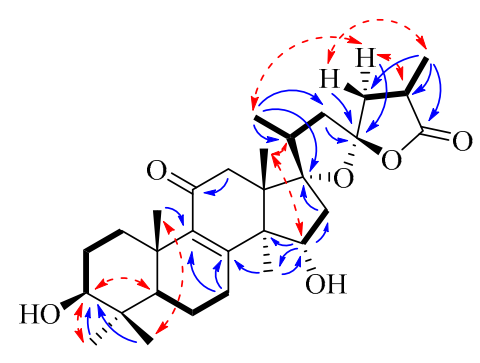

1
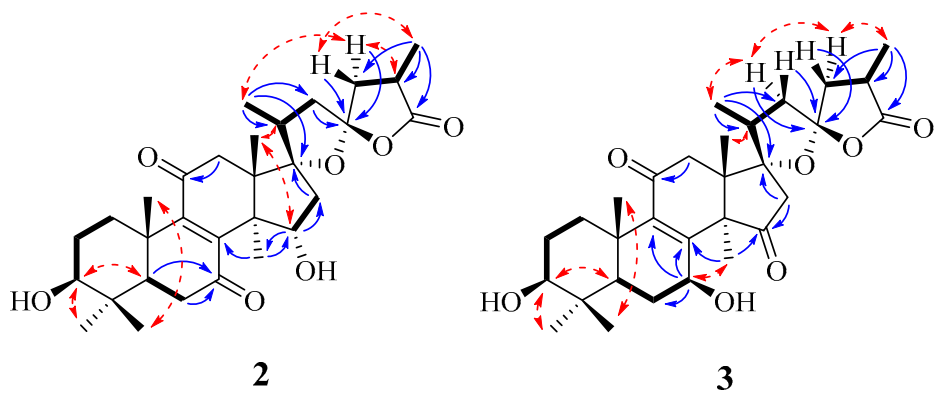

3

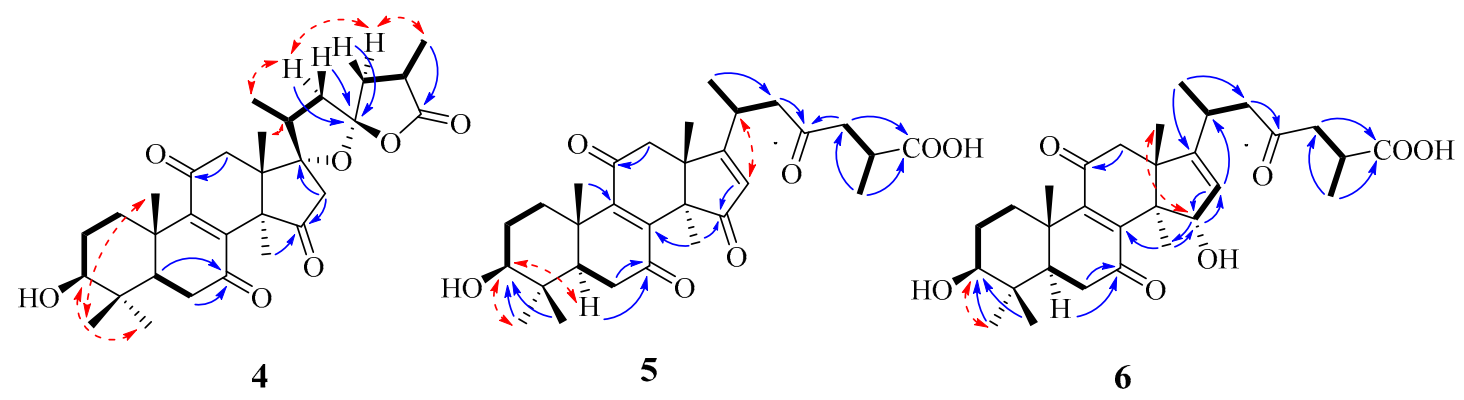

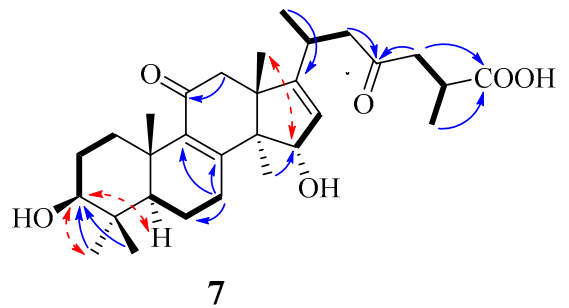

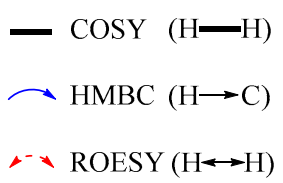

Figure 2. The key COSY, HMBC, and ROESY correlations of 1-7. Bold line (-): hydrogen-hydrogen correlation in the correlation spectroscopy (COSY) spectrum. One-way arrows $(\rightarrow)$ : heteronuclear multiple bond correlation from hydrogen to carbon in the heteronuclear multiple bond correlation (HMBC) spectrum. Double sided arrows $(\leftrightarrow)$ : the correlations between hydrogen and hydrogen in the rotating-frame nuclear overhauser effect correlation spectroscopy (ROESY) spectrum. 
Table 1. ${ }^{1} \mathrm{H}\left(600 \mathrm{MHz}, \mathrm{CDCl}_{3}\right)$ and ${ }^{13} \mathrm{C} \mathrm{NMR}\left(150 \mathrm{MHz}, \mathrm{CDCl}_{3}\right)$ spectroscopic data for $\mathbf{1}-\mathbf{4}$ and $\mathbf{8}(\delta$ in ppm, $J$ in $\mathrm{Hz})$.

\begin{tabular}{|c|c|c|c|c|c|c|c|c|c|c|}
\hline \multirow[b]{2}{*}{ No } & \multicolumn{2}{|r|}{$1^{\mathrm{a}}$} & \multicolumn{2}{|r|}{2} & \multicolumn{2}{|r|}{3} & \multicolumn{2}{|r|}{4} & \multicolumn{2}{|r|}{8} \\
\hline & $\delta_{\mathrm{C}}$ & $\delta_{\mathrm{H}}$ & $\delta_{\mathrm{C}}$ & $\delta_{\mathrm{H}}$ & $\delta_{\mathrm{C}}$ & $\delta_{\mathrm{H}}$ & $\delta_{\mathrm{C}}$ & $\delta_{\mathrm{H}}$ & $\delta_{\mathrm{C}}$ & $\delta_{\mathrm{H}}$ \\
\hline 1 & $35.21, \mathrm{CH}_{2}$ & $\begin{array}{c}1.36, \mathrm{dt}(13.2,3.0) ; \\
3.51 \mathrm{~m}\end{array}$ & 34.1, $\mathrm{CH}_{2}$ & $1.24, \mathrm{~m} ; 2.83, \mathrm{dt}(13.2,3.0)$ & $34.7, \mathrm{CH}_{2}$ & $\begin{array}{l}0.97 \mathrm{dd}(13.2,4.2) \\
2.83, \mathrm{dt}(13.2,3.6)\end{array}$ & 33.7, $\mathrm{CH}_{2}$ & $\begin{array}{l}\text { 1.34, } \mathrm{dt}(13.8,3.6) \\
2.84, \mathrm{dt}(13.8,3.6)\end{array}$ & $34.5, \mathrm{CH}_{2}$ & $\begin{array}{c}0.92, \mathrm{~m} ; \\
2.75, \mathrm{dt}(13.8,3.6)\end{array}$ \\
\hline 2 & 29.0, $\mathrm{CH}_{2}$ & $\begin{array}{c}1.99, \mathrm{~m} ; \\
2.06, \mathrm{dt}(13.8,3.0)\end{array}$ & $27.5, \mathrm{CH}_{2}$ & $\begin{array}{l}1.70, \mathrm{dd}(12.0,3.6) ; \\
1.76, \mathrm{~m}\end{array}$ & 27.7, $\mathrm{CH}_{2}$ & $1.67, \mathrm{~m}$ & $27.3, \mathrm{CH}_{2}$ & $\begin{array}{c}\text { 1.68, dt (12.0, 3.6); } \\
1.76, \mathrm{~m}\end{array}$ & $27.5, \mathrm{CH}_{2}$ & $\begin{array}{c}1.65, \mathrm{~m} ; \\
2.11, \mathrm{dd}(12.6,7.2)\end{array}$ \\
\hline 3 & $77.8, \mathrm{CH}$ & 3.52, dd overlapped & $77.3, \mathrm{CH}$ & $3.29, \mathrm{dd}(11.4,4.8)$ & $78.2, \mathrm{CH}$ & $3.22, \mathrm{dd}(11.4,4.8)$ & $77.5, \mathrm{CH}$ & $3.30, \mathrm{dd}(11.4,4.2)$ & $78.2, \mathrm{CH}$ & $3.23, \mathrm{dd}(11.4,4.8)$ \\
\hline 4 & $39.7, \mathrm{C}$ & & $38.7, \mathrm{C}$ & & $38.9, \mathrm{C}$ & & $39.0, \mathrm{C}$ & & $38.6, \mathrm{C}$ & \\
\hline 5 & $52.4, \mathrm{CH}$ & 1.18, br d $(12.0)$ & $49.3, \mathrm{CH}$ & $1.55, \mathrm{dd}(9.6,7.8)$ & $49.0, \mathrm{CH}$ & 0.88, br d $(12.0)$ & $49.9, \mathrm{CH}$ & 1.63, dd overlapped & $48.9, \mathrm{CH}$ & $0.92, \mathrm{~d}(12.0)$ \\
\hline 6 & $17.9, \mathrm{CH}_{2}$ & $1.51, \mathrm{~m} ; 1.84, \mathrm{~m}$ & $36.2, \mathrm{CH}_{2}$ & $2.56, \mathrm{~m} ; 2.57, \mathrm{~m}$ & 26.6, $\mathrm{CH}_{2}$ & $\begin{array}{l}\text { 1.59, } \mathrm{dt}(13.2,3.6) ; \\
2.19, \mathrm{dd}(13.2,8.4)\end{array}$ & $35.9, \mathrm{CH}_{2}$ & $2.58, \mathrm{~m} ; 2.62, \mathrm{~m}$ & 27.7, $\mathrm{CH}_{2}$ & $1.61, \mathrm{~m} ; 1.65, \mathrm{~m}$ \\
\hline 7 & $30.7, \mathrm{CH}_{2}$ & $\begin{array}{l}2.76, \mathrm{dd}(21.0,5.4) ; \\
\text { 2.96, } \mathrm{m}\end{array}$ & 205.0,C & & $66.7, \mathrm{CH}$ & $4.81, \mathrm{~m}$ & $198.5, \mathrm{C}$ & & 69.0, CH & 4.60, br t (6.6) \\
\hline 8 & $165.2, \mathrm{C}$ & & $150.3, \mathrm{C}$ & & $157.5, \mathrm{C}$ & & $148.9, \mathrm{C}$ & & $158.7, \mathrm{C}$ & \\
\hline 9 & $139.9, \mathrm{C}$ & & $154.5, \mathrm{C}$ & & $142.4, \mathrm{C}$ & & $151.5, \mathrm{C}$ & & $141.6, \mathrm{C}$ & \\
\hline 10 & $38.4, \mathrm{C}$ & & $40.0, \mathrm{C}$ & & $38.6, \mathrm{C}$ & & $40.4, \mathrm{C}$ & & $38.5, \mathrm{C}$ & \\
\hline 11 & 198.6, C & & $201.8, \mathrm{C}$ & & 198.1, C & & $200.2, \mathrm{C}$ & & $200.2, \mathrm{C}$ & \\
\hline 12 & 47.4, $\mathrm{CH}_{2}$ & $\begin{array}{l}\text { 2.46, d (16.8); } \\
\text { 3.38, d (16.8) }\end{array}$ & $47.2, \mathrm{CH}_{2}$ & $\begin{array}{l}\text { 2.35, d (16.8); } \\
3.25, \mathrm{~d}(16.8)\end{array}$ & $44.1, \mathrm{CH}_{2}$ & $\begin{array}{l}\text { 2.48, d (16.8); } \\
3.17, \mathrm{~d}(16.8)\end{array}$ & $44.7, \mathrm{CH}_{2}$ & $\begin{array}{l}\text { 2.48, d (16.2); } \\
3.20, \mathrm{~d}(16.2)\end{array}$ & 47.0, $\mathrm{CH}_{2}$ & $\begin{array}{l}\text { 2.27, d (15.0); } \\
3.12, \mathrm{~d}(15.0)\end{array}$ \\
\hline 13 & $50.2, \mathrm{C}$ & & $50.7 \mathrm{C}$ & & $48.1, \mathrm{C}$ & & $47.3, \mathrm{C}$ & & $49.5, \mathrm{C}$ & \\
\hline 14 & $54.4, \mathrm{C}$ & & $53.5, \mathrm{C}$ & & $59.1, \mathrm{C}$ & & $56.5, \mathrm{C}$ & & $54.3, \mathrm{C}$ & \\
\hline 15 & 72.9, $\mathrm{CH}$ & $4.72, \mathrm{dd}(9.0,7.2)$ & $72.7, \mathrm{CH}$ & $4.45, \mathrm{dd}(9.0,7.2)$ & $216.1, \mathrm{C}$ & & $207.1, \mathrm{C}$ & & 73.0, CH & $4.80, \mathrm{t}(8.4)$ \\
\hline 16 & 47.3, $\mathrm{CH}_{2}$ & $\begin{array}{l}2.52, \mathrm{dd}(15.0,9.0) ; \\
2.80, \mathrm{dd}(15.0,7.2)\end{array}$ & 44.3, $\mathrm{CH}_{2}$ & $\begin{array}{l}\text { 2.32, dd (15.6, 9.0); } \\
2.53, \mathrm{dd}(15.6,7.2)\end{array}$ & 48.0, $\mathrm{CH}_{2}$ & $\begin{array}{l}\text { 2.47, d (20.4); } \\
3.35, \mathrm{~d}(20.4)\end{array}$ & $48.1, \mathrm{CH}_{2}$ & $\begin{array}{l}\text { 2.45, d (16.2); } \\
3.33, \mathrm{~d}(16.2)\end{array}$ & $44.7, \mathrm{CH}_{2}$ & $\begin{array}{c}2.29, \mathrm{~m} ; \\
2.41, \mathrm{dd}(15.0,8.4) \\
\end{array}$ \\
\hline 17 & $95.6, \mathrm{C}$ & & $94.7, \mathrm{C}$ & & $91.8, \mathrm{C}$ & & $92.2, \mathrm{C}$ & & $94.7, \mathrm{C}$ & \\
\hline 18 & $19.8, \mathrm{CH}_{3}$ & $1.15, \mathrm{~s}$ & $20.0, \mathrm{CH}_{3}$ & $1.05, \mathrm{~s}$ & $20.9, \mathrm{CH}_{3}$ & $1.18, \mathrm{~s}$ & $20.0, \mathrm{CH}_{3}$ & $1.03, \mathrm{~s}$ & $19.7, \mathrm{CH}_{3}$ & $1.16, \mathrm{~s}$ \\
\hline 19 & $19.4, \mathrm{CH}_{3}$ & $1.42, \mathrm{~s}$ & $17.4, \mathrm{CH}_{3}$ & $1.29, \mathrm{~s}$ & $18.3, \mathrm{CH}_{3}$ & $1.21, \mathrm{~s}$ & $17.7, \mathrm{CH}_{3}$ & $1.20, \mathrm{~s}$ & $19.4, \mathrm{CH}_{3}$ & $1.26, \mathrm{~s}$ \\
\hline 20 & $43.7, \mathrm{CH}$ & $2.14, \mathrm{~m}$ & $43.6, \mathrm{CH}$ & $2.26, \mathrm{~m}$ & $43.1, \mathrm{CH}$ & $2.38, \mathrm{~m}$ & $43.3, \mathrm{CH}$ & $2.38, \mathrm{~m}$ & $48.3, \mathrm{CH}$ & $2.29, \mathrm{~m}$ \\
\hline 21 & $17.9, \mathrm{CH}_{3}$ & $0.91, \mathrm{~d}(6.6)$ & $18.1, \mathrm{CH}_{3}$ & $1.01, \mathrm{~d}(6.6)$ & $18.2, \mathrm{CH}_{3}$ & $1.12, \mathrm{~d}(6.6)$ & $18.3, \mathrm{CH}_{3}$ & $1.10, \mathrm{~d}(7.2)$ & $18.1, \mathrm{CH}_{3}$ & $1.02, \mathrm{~d}(7.2)$ \\
\hline 22 & $44.61, \mathrm{CH}_{2}$ & $\begin{array}{c}1.76 \mathrm{~d}(13.8) ; \\
2.64, \mathrm{dd}(13.8,6.6)\end{array}$ & 44.6, $\mathrm{CH}_{2}$ & $\begin{array}{c}1.81, \mathrm{~d}(13.8) \\
2.73, \mathrm{dd}(13.8,6.6)\end{array}$ & 44.4, $\mathrm{CH}_{2}$ & $\begin{array}{c}1.93, \mathrm{~d}(14.4) ; \\
2.73, \mathrm{dd}(14.4,7.2)\end{array}$ & $44.3, \mathrm{CH}_{2}$ & $\begin{array}{c}1.91, \mathrm{~d}(14.4) ; \\
2.73, \mathrm{dd}(14.4,6.6)\end{array}$ & $44.6, \mathrm{CH}_{2}$ & $\begin{array}{c}\text { 1.84, d (14.4); } \\
2.72 \text {, dd (14.4, 6.6) }\end{array}$ \\
\hline 23 & 113.3, C & & 112.7, C & & 113.0, C & & $112.9, \mathrm{C}$ & & 113.3, C & \\
\hline 24 & $44.62, \mathrm{CH}_{2}$ & $\begin{array}{c}1.96, \mathrm{~d}(12.6) ; \\
2.36, \mathrm{dd}(12.6,8.4)\end{array}$ & $44.9, \mathrm{CH}_{2}$ & $\begin{array}{l}2.03, \mathrm{dd}(12.6,11.4) ; \\
2.47, \mathrm{dd}(12.6,8.4)\end{array}$ & $44.5, \mathrm{CH}_{2}$ & $\begin{array}{l}2.10, \mathrm{dd}(13.2,11.4) ; \\
2.57, \mathrm{dd}(13.2,8.4)\end{array}$ & 44.6, $\mathrm{CH}_{2}$ & $\begin{array}{l}\text { 2.09, dd }(12.6,11.4) ; \\
\text { 2.56, dd overlapped }\end{array}$ & $44.7, \mathrm{CH}_{2}$ & $\begin{array}{l}2.05, \mathrm{dd}(12.6,11.4) ; \\
2.49, \mathrm{dd}(12.6,8.4)\end{array}$ \\
\hline 25 & $35.7, \mathrm{CH}$ & $3.02, \mathrm{~m}$ & $35.4, \mathrm{CH}$ & $2.92, \mathrm{~m}$ & $35.4, \mathrm{CH}$ & $2.92, \mathrm{~m}$ & $35.3, \mathrm{CH}$ & $2.94, \mathrm{~m}$ & $35.5, \mathrm{CH}$ & $2.96, \mathrm{~m}$ \\
\hline 26 & 178.7,C & & $178.5, \mathrm{C}$ & & $178.3, \mathrm{C}$ & & 178.2, C & & $179.2, \mathrm{C}$ & \\
\hline 27 & $15.1, \mathrm{CH}_{3}$ & $1.23, \mathrm{~d}(7.2)$ & $14.9, \mathrm{CH}_{3}$ & $1.25, \mathrm{~d}(6.6)$ & $14.9, \mathrm{CH}_{3}$ & $1.29, \mathrm{~d}(7.2)$ & $14.9, \mathrm{CH}_{3}$ & $1.28, \mathrm{~d}(7.2)$ & $14.9, \mathrm{CH}_{3}$ & $1.28, \mathrm{~d}(7.2)$ \\
\hline 28 & $28.9, \mathrm{CH}_{3}$ & $1.27, \mathrm{~s}$ & $27.7, \mathrm{CH}_{3}$ & $1.03, \mathrm{~s}$ & $28.2, \mathrm{CH}_{3}$ & $1.04, \mathrm{~s}$ & $27.7, \mathrm{CH}_{3}$ & $1.03, \mathrm{~s}$ & $28.1, \mathrm{CH}_{3}$ & $1.03, \mathrm{~s}$ \\
\hline 29 & $16.8, \mathrm{CH}_{3}$ & $1.13, \mathrm{~s}$ & $15.4, \mathrm{CH}_{3}$ & $0.89, \mathrm{~s}$ & $15.5, \mathrm{CH}_{3}$ & $0.87, \mathrm{~s}$ & $15.4, \mathrm{CH}_{3}$ & $0.89, \mathrm{~s}$ & $15.7, \mathrm{CH}_{3}$ & $0.85, \mathrm{~s}$ \\
\hline 30 & $21.8, \mathrm{CH}_{3}$ & $1.68, \mathrm{~s}$ & $22.6, \mathrm{CH}_{3}$ & $1.24, \mathrm{~s}$ & $26.9, \mathrm{CH} 3$ & $1.41, \mathrm{~s}$ & $25.8, \mathrm{CH}_{3}$ & $1.54, \mathrm{~s}$ & $21.2, \mathrm{CH}_{3}$ & $1.33, \mathrm{~s}$ \\
\hline
\end{tabular}

${ }^{\text {a }}$ Recorded in pyridine- $d_{5}$. 
Compound 3 was isolated as a white powder. Its molecular formula was defined as $\mathrm{C}_{30} \mathrm{H}_{42} \mathrm{O}_{7}$ by the HRESIMS ion peak at $m / z 513.2855[\mathrm{M}-\mathrm{H}]^{-}$(calcd. for $\mathrm{C}_{30} \mathrm{H}_{41} \mathrm{O}_{7}, 513.2852$ ). The presence of hydroxy $\left(3474 \mathrm{~cm}^{-1}\right)$, carbonyl $\left(1774\right.$ and $\left.1750 \mathrm{~cm}^{-1}\right)$, and $\alpha, \beta$-unsaturated carbonyl $\left(1656 \mathrm{~cm}^{-1}\right.$ and $255 \mathrm{~nm}$ ) groups was confirmed by the IR and UV spectra. The ${ }^{1} \mathrm{H}$ NMR, ${ }^{13} \mathrm{C}$ NMR (Table 1), and HSQC spectra showed the presence of seven methyls $\left[\delta_{\mathrm{H}} 0.87(3 \mathrm{H}, \mathrm{s}), 1.04(3 \mathrm{H}, \mathrm{s}), 1.12(3 \mathrm{H}, \mathrm{d}), 1.18\right.$ $(3 \mathrm{H}, \mathrm{s}), 1.21(3 \mathrm{H}, \mathrm{s}), 1.29(3 \mathrm{H}, \mathrm{d})$, and $1.41(3 \mathrm{H}, \mathrm{s})]$, seven methylenes, five methines including two oxygenated methine groups $\delta_{\mathrm{C}} 66.7$ and $72.7, \mathrm{six} \mathrm{sp}^{3}$ quaternary carbons including two oxygenated quaternary carbons at $\delta_{\mathrm{C}} 91.8$ and 113.0, one tetrasubstituted olefinic group ( $\delta_{\mathrm{C}} 142.4$ and 157.5), and three carbonyl groups $\left(\delta_{\mathrm{C}} 198.1,216.1\right.$, and 178.3). The 1D NMR spectroscopic data indicated the gross structure of $\mathbf{3}$ was same as that of $\mathbf{2}$. The differences were that an oxygenated methine carbon signal $\left(\delta_{C}\right.$ 66.7) resonated at higher field and a ketone carbon signal $\left(\delta_{C} 216.1\right)$ at lower field in 3 compared with those of 2 . The HMBC correlations from $\mathrm{H}-7\left(\delta_{\mathrm{H}} 4.81\right)$ to C-8 $\left(\delta_{\mathrm{C}} 157.5\right)$ and C-9 $\left(\delta_{\mathrm{C}} 142.4\right)$, combined with the ${ }^{1} \mathrm{H}-{ }^{1} \mathrm{H}$ COSY correlations of $\mathrm{H}-7\left(\delta_{\mathrm{H}} 4.81\right)$ with $\mathrm{H}-6\left(\delta_{\mathrm{H}}\right.$ 2.18 and 1.59), confirmed the presence of $7-\mathrm{OH}$. The ketone group at $\mathrm{C}-15$ was supported by the HMBC correlations from $\mathrm{H}-30\left(\delta_{\mathrm{H}} 1.41\right)$ and $\mathrm{H}-16\left(\delta_{\mathrm{H}} 3.35\right.$ and 2.47$)$ to $\mathrm{C}-15\left(\delta_{\mathrm{C}} 216.1\right)$. The ROESY correlation of $\mathrm{H}-7\left(\delta_{\mathrm{H}} 4.81\right)$ with $\mathrm{H}-30\left(\delta_{\mathrm{H}} 1.41\right)$ assigned $7-\mathrm{OH}$ as having $\beta$-orientation. The relative configurations of Me-21 and Me-27 were all determined to be $\alpha$-orientation on the basis of the ROESY correlations of H-18 $\left(\delta_{\mathrm{H}} 1.18\right)$ with $\mathrm{H}-20\left(\delta_{\mathrm{H}} 2.38\right), \mathrm{H}-22 \alpha\left(\delta_{\mathrm{H}} 1.93\right)$ with $\mathrm{H}-21\left(\delta_{\mathrm{H}} 1.12\right)$ and $\mathrm{H}-24 \alpha\left(\delta_{\mathrm{H}} 1.93\right)$, and $\mathrm{H}-24 \alpha\left(\delta_{\mathrm{H}} 1.93\right)$ with $\mathrm{H}-27\left(\delta_{\mathrm{H}} 1.29\right)$. Therefore, compound 3 was assigned as $(17 S, 23 S)$-17,23-epoxy-3 $\beta, 7 \beta$-dihydroxy-11,15-dioxo-5 $\alpha$-lanosta-8-en-26,23-olide.

Compound 4 was obtained as a white powder. Its molecular formula was established as $\mathrm{C}_{30} \mathrm{H}_{40} \mathrm{O}_{7}$ by the HRESIMS ion peak at $\mathrm{m} / z 511.2700[\mathrm{M}-\mathrm{H}]^{-}$(calcd. for $\mathrm{C}_{30} \mathrm{H}_{39} \mathrm{O}_{7}, 511.2696$ ). The IR and UV spectra displayed the presence of hydroxy $\left(3490 \mathrm{~cm}^{-1}\right)$, carbonyl $\left(1774\right.$ and $\left.1750 \mathrm{~cm}^{-1}\right)$, and $\alpha, \beta$-unsaturated carbonyl $\left(1672 \mathrm{~cm}^{-1}\right.$ and $\left.265 \mathrm{~nm}\right)$ groups. A comparison of its NMR spectroscopic data with those of $\mathbf{3}$ showed their structural similarities. The only difference was the presence of ketone at C-7 in 4 instead of the hydroxy group. The ketone group was located at C-7 due to the key HMBC correlations of $\mathrm{H}-6\left(\delta_{\mathrm{H}} 2.62\right.$ and 2.58$)$ and $\mathrm{H}-5\left(\delta_{\mathrm{H}} 1.63\right)$ with $\mathrm{C}-7\left(\delta_{\mathrm{C}} 198.5\right)$. The relative configuration for 4 was determined based on the ROESY experiment and comparison of NMR spectroscopic data with those of 3. The ROESY correlations of H-3 $\left(\delta_{\mathrm{H}} 3.30\right)$ with $\mathrm{H}-28\left(\delta_{\mathrm{H}} 1.03\right), \mathrm{H}-20\left(\delta_{\mathrm{H}} 2.38\right)$ with $\mathrm{H}-18\left(\delta_{\mathrm{H}}\right.$ $1.03), \mathrm{H}-22 \alpha\left(\delta_{\mathrm{H}} 1.91\right)$ with $\mathrm{H}-21\left(\delta_{\mathrm{H}} 1.10\right)$ and $\mathrm{H}-24 \alpha\left(\delta_{\mathrm{H}} 2.09\right)$, and $\mathrm{H}-24 \alpha\left(\delta_{\mathrm{H}} 2.09\right)$ with $\mathrm{H}-27\left(\delta_{\mathrm{H}} 1.28\right)$ assigned 3-OH, Me-21, and Me-27, were $\beta-, \alpha-$, and $\alpha$-orientation, respectively. Therefore, compound 4 was established as (17S,23S)-17,23-epoxy-3 $\beta$-hydroxy-7,11,15-trioxo-5 $\alpha$-lanosta-8-en-26,23-olide.

Compound 5 was obtained as a white powder. Its molecular formula was determined to be $\mathrm{C}_{30} \mathrm{H}_{40} \mathrm{O}_{7}$ based on the HRESIMS peak ion at $m / z 511.2684[\mathrm{M}-\mathrm{H}]^{-}$(calcd. for $\mathrm{C}_{30} \mathrm{H}_{39} \mathrm{O}_{7}, 511.2696$ ). The ${ }^{1} \mathrm{H}$ NMR, ${ }^{13} \mathrm{C}$ NMR (Table 2), and HSQC spectra indicated that 5 was lanostane ganoderic acid closely similar to ganoderesin C [14]. The only difference was that 5 possessed a tetrasubstituted olefinic group between C-8 and C-9 whereas ganoderesin $C$ did not. The tetrasubstituted olefinic group was established using $\mathrm{HMBC}$ correlations of $\mathrm{H}-30\left(\delta_{\mathrm{H}} 1.39\right)$ with $\mathrm{C}-8\left(\delta_{\mathrm{C}} 151.5\right)$, and $\mathrm{H}-19$ $\left(\delta_{\mathrm{H}} 1.08\right)$ with C-9 $\left(\delta_{\mathrm{C}} 154.0\right)$. The planar structure of 5 was confirmed by the $\mathrm{HMBC}$ and ${ }^{1} \mathrm{H}-{ }^{1} \mathrm{H}$ COSY correlations (Figure 2). The ROESY correlations of H-3 $\left(\delta_{\mathrm{H}} 3.18\right)$ with $\mathrm{H}-28\left(\delta_{\mathrm{H}} 0.96\right)$ and $\mathrm{H}-5\left(\delta_{\mathrm{H}} 1.71\right)$ assigned $3-\mathrm{OH}$ as $\beta$-orientation. Therefore, compound 5 was elucidated as $3 \beta$-hydroxy-7,11,15,23-tetraoxo-5 $\alpha$-lanosta-8,16-dien-26-oic acid. 
Table 2. ${ }^{1} \mathrm{H}\left(600 \mathrm{MHz}, \mathrm{CD}_{3} \mathrm{OD}\right)$ and ${ }^{13} \mathrm{C}\left(150 \mathrm{MHz}, \mathrm{CD}_{3} \mathrm{OD}\right) \mathrm{NMR}$ spectroscopic data for 5-7 ( $\delta$ in ppm, $J$ in $\mathrm{Hz}$ ).

\begin{tabular}{|c|c|c|c|c|c|c|}
\hline \multirow[b]{2}{*}{ No } & \multicolumn{2}{|r|}{5} & \multicolumn{2}{|r|}{6} & \multicolumn{2}{|r|}{7} \\
\hline & $\delta_{\mathrm{C}}$ & $\delta_{\mathrm{H}}$ & $\delta_{\mathrm{C}}$ & $\delta_{\mathrm{H}}$ & $\delta_{\mathrm{C}}$ & $\delta_{\mathrm{H}}$ \\
\hline 1 & $35.2, \mathrm{CH}_{2}$ & $\begin{array}{c}1.37, \mathrm{dd}(13.2,4.2) \\
2.85, \mathrm{~m}\end{array}$ & $35.4, \mathrm{CH}_{2}$ & $\begin{array}{c}1.29, \mathrm{~m} ; 2.92, \mathrm{dt} \\
(13.8,3.6)\end{array}$ & $35.8, \mathrm{CH}_{2}$ & $\begin{array}{l}\text { 1.08, dd (13.2, 3.6); } \\
\text { 2.99, dt }(13.2,3.6)\end{array}$ \\
\hline 2 & $28.1, \mathrm{CH}_{2}$ & $1.62, \mathrm{~m}$ & $28.6, \mathrm{CH}_{2}$ & $1.69, \mathrm{~m} ; 1.74, \mathrm{~m}$ & $28.6, \mathrm{CH}_{2}$ & $1.62, \mathrm{~m} ; 1.68, \mathrm{~m}$ \\
\hline 3 & $78.4, \mathrm{CH}$ & $3.18, \mathrm{dd}(11.4,4.8)$ & $78.2, \mathrm{CH}$ & $3.22, \mathrm{dd}(11.4,4.8)$ & $79.5, \mathrm{CH}$ & 3.17, dd $(11.4,4.8)$ \\
\hline 4 & $40.1, \mathrm{C}$ & & $40.1, C$ & & $40.3, \mathrm{C}$ & \\
\hline 5 & $50.5, \mathrm{CH}$ & $1.71, \mathrm{dd}(13.8,4.2)$ & $51.5, \mathrm{CH}$ & $1.58, \mathrm{dd}(15.0,1.8)$ & $53.7, \mathrm{CH}$ & $0.95, \mathrm{~d}(13.2)$ \\
\hline 6 & 36.0, $\mathrm{CH}_{2}$ & $\begin{array}{c}\text { 2.49, d (13.8); } \\
\text { 2.55, dd }(13.8,4.2)\end{array}$ & $36.8, \mathrm{CH}_{2}$ & $2.55, \mathrm{~m} ; 2.70, \mathrm{t}(15.0)$ & 18.7, $\mathrm{CH}_{2}$ & $1.51, \mathrm{~m} ; 1.81, \mathrm{~m}$ \\
\hline 7 & $200.3, C$ & & 206.3, C & & $32.9, \mathrm{CH}_{2}$ & $2.55, \mathrm{~m}$ \\
\hline 8 & $151.5, C$ & & $150.9, \mathrm{C}$ & & $166.5, \mathrm{C}$ & \\
\hline 9 & 154.0, C & & $155.94, \mathrm{C}$ & & $140.9, \mathrm{C}$ & \\
\hline 10 & $42.0, \mathrm{C}$ & & $41.8, \mathrm{C}$ & & $39.4, \mathrm{C}$ & \\
\hline 11 & $200.8, C$ & & $202.6, C$ & & $201.2, \mathrm{C}$ & \\
\hline 12 & $45.6, \mathrm{CH}_{2}$ & $\begin{array}{l}\text { 2.54, d (16.8); } \\
3.09, \mathrm{~d}(16.8)\end{array}$ & $48.9, \mathrm{CH}_{2}$ & $\begin{array}{l}\text { 2.42, d (16.2); } \\
\text { 3.08, d (16.2) }\end{array}$ & $48.7, \mathrm{CH}_{2}$ & $\begin{array}{l}\text { 2.24, d (16.8); } \\
2.94, \mathrm{~d}(16.8)\end{array}$ \\
\hline 13 & $53.2, \mathrm{C}$ & & $53.8, \mathrm{C}$ & & $53.4, \mathrm{C}$ & \\
\hline 14 & $56.6, \mathrm{C}$ & & $55.3, \mathrm{C}$ & & $58.0, \mathrm{C}$ & \\
\hline 15 & 206.7, C & & $78.4, \mathrm{CH}$ & $4.95, \mathrm{br} \mathrm{s}$ & $77.8, \mathrm{CH}$ & $4.90, \mathrm{br} \mathrm{s}$ \\
\hline 16 & 124.0, $\mathrm{CH}$ & $5.65, \mathrm{~s}$ & $124.5, \mathrm{CH}$ & $5.26, \mathrm{~s}$ & $125.7, \mathrm{CH}$ & $5.18, \mathrm{~s}$ \\
\hline 17 & $186.7, \mathrm{C}$ & & $155.98, \mathrm{C}$ & & $156.3, \mathrm{C}$ & \\
\hline 18 & $30.1, \mathrm{CH}_{3}$ & $1.02, \mathrm{~s}$ & $23.3, \mathrm{CH}_{3}$ & $0.95, \mathrm{~s}$ & 23.0, $\mathrm{CH}_{3}$ & $0.96, \mathrm{~s}$ \\
\hline 19 & $17.7, \mathrm{CH}_{3}$ & $1.08, \mathrm{~s}$ & $17.7, \mathrm{CH}_{3}$ & $1.30, \mathrm{~s}$ & $19.4, \mathrm{CH}_{3}$ & $1.12, \mathrm{~s}$ \\
\hline 20 & $30.7, \mathrm{CH}$ & $2.93, \mathrm{~m}$ & $28.5, \mathrm{CH}$ & $2.63, \mathrm{~m}$ & $28.9, \mathrm{CH}$ & $2.62, \mathrm{~m}$ \\
\hline 21 & $20.1, \mathrm{CH}_{3}$ & $1.05, \mathrm{~d}(6.6)$ & 21.0, $\mathrm{CH}_{3}$ & $1.04, \mathrm{~d}(6.6)$ & 21.0, $\mathrm{CH}_{3}$ & $1.04, \mathrm{~d}(7.2)$ \\
\hline 22 & $48.5, \mathrm{CH}_{2}$ & $\begin{array}{c}\text { 2.70, dd (17.4, 5.4); } \\
2.88, \mathrm{~d}(17.4)\end{array}$ & $49.4, \mathrm{CH}_{2}$ & $\begin{array}{c}2.57, \mathrm{~d}(16.8) ; \\
2.77, \mathrm{dd}(16.8,7.2)\end{array}$ & $49.5, \mathrm{CH}_{2}$ & $\begin{array}{c}\text { 2.54, d (16.8); } \\
2.73, \mathrm{dd}(16.8,7.2)\end{array}$ \\
\hline 23 & 209.0, C & & $209.9, C$ & & $210.0, C$ & \\
\hline 24 & 47.3, $\mathrm{CH}_{2}$ & $\begin{array}{l}2.49, \mathrm{~m} ; \\
2.81, \mathrm{~m}\end{array}$ & $47.5, \mathrm{CH}_{2}$ & $\begin{array}{l}2.53, \mathrm{~m} \\
2.85, \mathrm{~m}\end{array}$ & $47.5, \mathrm{CH}_{2}$ & $\begin{array}{l}\text { 2.52, dd }(13.2,8.4) ; \\
2.85 \text {, dd }(13.2,9.0)\end{array}$ \\
\hline 25 & $36.2, \mathrm{CH}$ & $2.78, \mathrm{~m}$ & $36.0, \mathrm{CH}$ & $2.82, \mathrm{~m}$ & $36.1, \mathrm{CH}$ & $2.83, \mathrm{~m}$ \\
\hline 26 & 179.6, C & & $179.9, \mathrm{C}$ & & 179.8, C & \\
\hline 27 & $17.7, \mathrm{CH}_{3}$ & $1.09, \mathrm{~d}(7.2)$ & $17.7, \mathrm{CH}_{3}$ & $1.14, \mathrm{~d}(7.2)$ & $17.7, \mathrm{CH}_{3}$ & $1.15, \mathrm{~d}(6.6)$ \\
\hline 28 & $28.3, \mathrm{CH}_{3}$ & $0.96, \mathrm{~s}$ & $28.3, \mathrm{CH}_{3}$ & $1.00, \mathrm{~s}$ & $29.1, \mathrm{CH}_{3}$ & $1.02, \mathrm{~s}$ \\
\hline 29 & $16.0, \mathrm{CH}_{3}$ & $0.83, \mathrm{~s}$ & $16.2, \mathrm{CH}_{3}$ & $0.89, \mathrm{~s}$ & 16.6, $\mathrm{CH}_{3}$ & $0.82, \mathrm{~s}$ \\
\hline 30 & $33.6, \mathrm{CH}_{3}$ & $1.39, \mathrm{~s}$ & $23.2, \mathrm{CH}_{3}$ & $1.17, \mathrm{~s}$ & $22.7, \mathrm{CH}_{3}$ & $1.21, \mathrm{~s}$ \\
\hline
\end{tabular}

Compound 6 was isolated as a white powder. Its molecular formula was assigned as $\mathrm{C}_{30} \mathrm{H}_{42} \mathrm{O}_{7}$ based on the HRESIMS ion peak at $m / z 513.2843[\mathrm{M}-\mathrm{H}]^{-}$(calcd. for $\mathrm{C}_{30} \mathrm{H}_{41} \mathrm{O}_{7}, 513.2852$ ). The ${ }^{1} \mathrm{H}$ NMR, ${ }^{13} \mathrm{C}$ NMR (Table 2), and HSQC spectra showed that 6 was lanostane-type triterpenoid closely similar to 5 . The only difference was an oxygenated methine group at C-15 in 6 instead of carbonyl group in 5. The key HMBC correlations from H-30 $\left(\delta_{\mathrm{H}} 1.17\right)$ to $\mathrm{C}-15\left(\delta_{\mathrm{C}} 78.4\right)$, and $\mathrm{H}-15\left(\delta_{\mathrm{H}} 4.95\right)$ to $\mathrm{C}-16\left(\delta_{\mathrm{C}} 124.5\right)$ and $\mathrm{C}-30\left(\delta_{\mathrm{C}} 23.2\right)$, combined with a ${ }^{1} \mathrm{H}-{ }^{1} \mathrm{H}$ COSY cross peak of $\mathrm{H}-15\left(\delta_{\mathrm{H}} 4.95\right)$ with $\mathrm{H}-16\left(\delta_{\mathrm{H}} 5.26\right)$, confirmed the presence of $15-\mathrm{OH}$. The $15-\mathrm{OH}$ was assigned as $\alpha$-orientation on the basis of the ROESY correlation of $\mathrm{H}-15\left(\delta_{\mathrm{H}} 4.95\right)$ with $\mathrm{H}-30\left(\delta_{\mathrm{H}} 1.17\right)$. Based on detail examination of $\mathrm{HMBC},{ }^{1} \mathrm{H}_{-}{ }^{1} \mathrm{H}$ COSY, and ROESY experiments, compound 6 was identified as $3 \beta, 15 \alpha$-dihydroxy-7,11,23-trioxo-5 $\alpha$-lanosta-8,16-dien-26-oic acid. 
Compound 7 was obtained as a white powder. Its molecular formula was determined to be $\mathrm{C}_{30} \mathrm{H}_{44} \mathrm{O}_{6}$ by the HRESIMS ion peak at $\mathrm{m} / z 499.3048[\mathrm{M}-\mathrm{H}]^{-}$(calcd. for $\mathrm{C}_{30} \mathrm{H}_{43} \mathrm{O}_{6}, 499.3060$ ). A comparison of NMR spectroscopic data (Table 2) with those of 6 indicated their structural similarities, except for the disappearance of one ketone group at C-7 in 7 . The methylene at C-7 was confirmed by the key HMBC correlations of $\mathrm{H}-7\left(\delta_{\mathrm{H}} 2.55\right)$ with C-6 $\left(\delta_{\mathrm{C}} 18.7\right), \mathrm{C}-8\left(\delta_{\mathrm{H}} 166.5\right)$, and C-9 $\left(\delta_{\mathrm{H}} 140.9\right)$, as well as COSY correlations of H-6 $\left(\delta_{\mathrm{H}} 1.81\right.$ and 1.51) with $\mathrm{H}-7\left(\delta_{\mathrm{H}} 2.55\right)$. Therefore, compound 7 was identified as $3 \beta, 15 \alpha$-dihydroxy-11,23-dioxo-5 $\alpha$-lanosta-8,16-dien-26-oic acid.

In addition, seven known compounds 8-14 were also isolated from G. resinaceum. By comparison of their NMR spectroscopic data and MS data with those reported in the literature, their structures

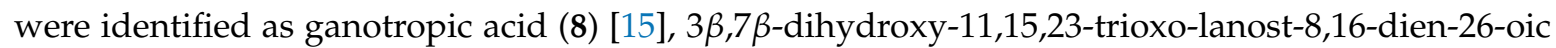
acid (9) [16], 3 $\beta, 7 \beta, 15 \beta$-trihydroxy-11,23-dioxo-lanost-8,16-dien-26-oic acid (10) [17], ganoderic acid $\mathrm{AM}_{1}$ (11) [18], ganoderic acid K (12) [19], ganoderic acid $\mathrm{C}_{2}$ (13) [19], and ganolucidic acid B (14) [20], respectively.

\subsection{Bioassay}

In an in vitro $\alpha$-glucosidase inhibitory assay, compounds 1-3, 5, 6, and 9-14 were evaluated for $\alpha$-glucosidase inhibitory activity. Compounds $\mathbf{1}$ and $\mathbf{2}$ exhibited more potent inhibitory activity against $\alpha$-glucosidase compared with the positive control drug acarbose $\left(\mathrm{IC}_{50}\right.$ value $2.76 \mathrm{mM}$ ) with $\mathrm{IC}_{50}$ value of $0.75 \pm 0.018 \mathrm{mM}$ and $1.64 \pm 0.022 \mathrm{mM}$, respectively. The inhibition rate of other compounds was less than $50 \%$ at the concentration of $3 \mathrm{mM}$ (Table S1 in Supplementary Material), suggesting that they showed no significant inhibitory activity against $\alpha$-glucosidase compared with acarbose; therefore, their $\mathrm{IC}_{50}$ value were not measured. The bioassay results are in line with the structure-activity relationships of $\alpha$-glucosidase inhibitory activity of triterpenoids, which was summarized in our previous report [3].

\section{Discussion}

Fourteen triterpenoids including seven new triterpenoids (1-7) and seven known analogues (8-14) were isolated from the fruiting bodies of G. resinaceum. The $\mathrm{IC}_{50}$ values of $\mathbf{1}$ and $\mathbf{2}$ are lower than that of the positive control drug acarbose, suggesting that $\mathbf{1}$ and $\mathbf{2}$ are strong $\alpha$-glucosidase inhibitors. Compounds 1-4 and $\mathbf{8}$ were determined to be triterpenoid lactones which possessed an oxaspirolactone moiety in the side chain, consisting of a five-membered ether ring, a five-membered lactone ring, and a characteristic C-23 spiro carbon. It is rare that such an oxaspirolactone moiety ring occurred in the lanostane-type triterpenoids. At present, no more than 40 lanostane-type triterpenoids isolated from natural products possessed such an oxaspirolactone moiety $[12,13,21]$. We proposed that compounds 1-4 and 8 were biogenetically derived from lanosterol derived by the mevalonic acid pathway. In the biosynthetic pathway (Figure 3), lanosterol undergoing multistep oxidation and double bond addition reactions would give key intermediate A, such as 11-14. Intermediate A would be further transformed into intermediate B (5-7, 9, and 10) via a reaction catalyzed by dehydrogenase. Intermediate B is oxidized via electrophilic addition reaction to generate intermediate $C$. The final and interesting phase of the biosynthesis involves the formation of the oxaspiro-E/F ring moiety through tandem cyclization. The hydroxy at C-17 attacks ketone via nucleophilic addition reaction, inducing tandem cyclization reaction to generate 1-4 and 8. Of note, Me-27 is $\alpha$ - or $\beta$-orientation in 1-4 and $\mathbf{8}$, this phenomenon allows us to tentatively hypothesize that the carboxylic group at C-26 was randomly attacked via nucleophilic addition reaction in the process of free rotation of side chain. Notably, compounds 1-4 and $\mathbf{8}$ provide unique insights into the biosynthetic pathway of lanostane-type triterpenoids in Ganoderma genus, for the formation of oxaspirolactone moiety involved the tandem cyclization reaction. 


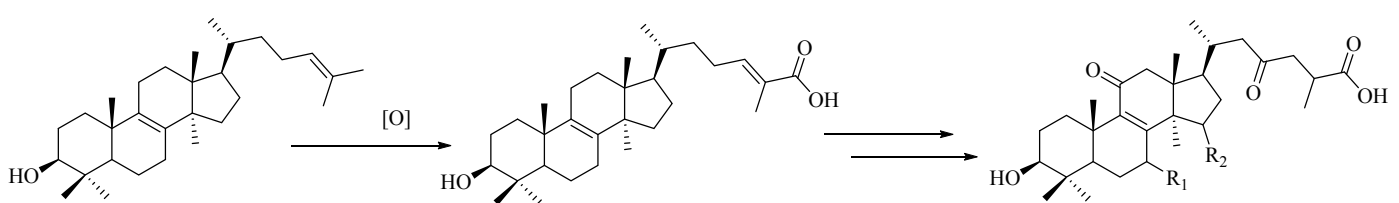

Lanosterol

ganoderma acid Z

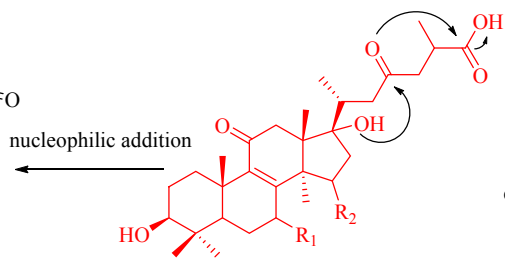

C
A

Dehydrogenase
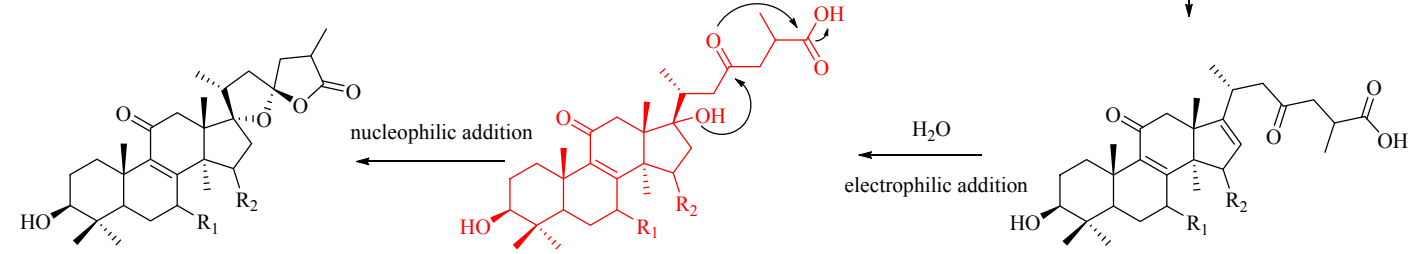

B

Figure 3. Plausible biogenetic pathway for 1-4 and 8. A, B, and C are supposed intermediates in the biosynthetic pathway of compounds 1-4 and 8. Intermediate A included compounds 11-14. Intermediate B included compounds 5-7, 9, and 10. At present, the representative compounds of intermediate $\mathbf{C}$ are not obtained from Ganoderma resinaceum.

\section{Materials and Methods}

\subsection{General Experimental Procedures}

NMR spectra data were recorded on a Bruker ascend 600 spectrometer (Bruker, Karlsruhe, Germany), with TMS used as a reference. All NMR samples were thermally equilibrated at $298 \mathrm{~K}$. The mixing time of ROESY spectrum is $300 \mathrm{~ms}$. The long-range coupling constant in HMBC experiment is $8.0 \mathrm{~Hz}$. Optical rotations were measured on PerkinElmer Model 341 polarimeter (PerkinElmer, Waltham, MA, USA). UV spectra data were acquired using HACH DR6000 UV-visible spectrophotometer (Hach, Loveland, CO, USA). IR spectra were recorded as KBr disks on PerkinElmer Spectrum 100 Series FT-IR spectrometers (PerkinElmer, Waltham, MA, USA). HRESIMS data were obtained on a LTQ Orbitrap XL ${ }^{\mathrm{TM}}$ Hybrid Ion Trap-Orbitrap FT-MS spectrometer (Thermo, Waltham, MA, USA). TLC was carried out on silica gel $\mathrm{GF}_{254}$ plates (Yantai Institute of Chemical Industry, Yantai, China) and spots were visualized by UV light ( 254 and/or $365 \mathrm{~nm}$ ) and spraying with $10 \% \mathrm{H}_{2} \mathrm{SO}_{4}$, followed by heating. Column chromatography was carried out using silica gel (Qingdao Marine Chemical Co. Ltd., Qingdao, China), MCI gel (CHP-20P, 75-150 $\mu \mathrm{m}$, Mitsubishi Chemical Corporation, Tokyo, Japan), ODS (35-70 $\mu \mathrm{m}$, Grace, Maryland, MD, USA), and Sephadex LH-20 (GE Healthcare Bio-Science AB, Uppsala, Sweden) as packing materials. Semipreparative HPLC was performed on a Shimadzu instrument (Shimadzu, Tokyo, Japan) coupled to CBM-20A system controller, LC-20AP pump, SPD-M20A Photodiode Array Detector, and SIL-10AP autosampler, and equipped with a Shimadzu PRC-ODS column $(250 \mathrm{~mm} \times 20 \mathrm{~mm}$ i.d., $15 \mu \mathrm{m})$. $\alpha$-Glucosidase type I (EC 3.2.1.20) from Saccharomyces cerevisiae and $p$-nitrophenyl $\alpha$-D-glucopyranoside (PNPG) were purchased from Sigma (St. Louis, MO, USA). Acarbose was purchased from Target Molecule Corp. (Boston, MA, USA). Absorbance was measured at $405 \mathrm{~nm}$ using a flexstation 3 multi-mode microplate reader (Molecular Devices, San Jose, CA, USA). Water bath heating was carried out in water bath (Memmert GmbH + Co. KG, Schwabach, Germany).

\subsection{Fungal Materials}

Fruiting bodies of Ganoderma resinaceum were purchased in December 2014 from Haikou Ruizhitang Wild Lingzhi Co., Ltd. (Hainan, China), and the sample has been collected in Changjiang 
(N 19.0066 ${ }^{\circ}, \mathrm{E} 109.1811^{\circ}$ ), Hainan province, China. The mushroom was identified by Shaoping Li, a corresponding author of the paper, based on the micro- and macro-morphology. A voucher specimen (No. ICMS-SQC-20141201) has been deposited at Institute of Chinese Medical Sciences, University of Macau.

\subsection{Extraction and Isolation}

The dried fruiting bodies of G. resinaceum ( $48 \mathrm{~kg}$ ) were powdered and then extracted with $95 \%$ $\mathrm{EtOH}(600 \mathrm{~L} \times 2 \mathrm{~h} \times 2)$ under reflux. The extract solution was concentrated under vacuum to afford the crude extract $(2.6 \mathrm{~kg})$. After removal of solvent, the extract was dispersed in water and partitioned with petroleum ether, EtOAc and $n-\mathrm{BuOH}$, successively. The HPLC and TLC profiling of EtOAc and $n$-BuOH extract showed their similarities in constituents, hence, the EtOAc extract was merged with the $n-\mathrm{BuOH}$ extract. The mixture of EtOAc and $n$-BuOH extract was subjected to silica gel column chromatography (CC) eluted with a gradient of $\mathrm{CHCl}_{3}-\mathrm{MeOH}$ solvent system $(100: 0-0: 100, v / v)$ to obtain three fractions (WE1-WE3).

WE1 (358 g) was separated on silica gel CC eluted with petroleum ether-acetone (100:0-0:100, $v / v$ ) to obtain four subfractions (WE11-WE14). WE12 (135 g) was chromatographed over silica gel CC eluted with petroleum ether-EtOAc (10:1-0:1, v/v) to yield fractions WE12A and WE12B. WE12B (55 g) was subjected to MCI gel CC eluted with $\mathrm{MeOH}-\mathrm{H}_{2} \mathrm{O}(70: 30-100: 0, v / v)$ to yield seven fractions (WE12B1-WB12B7). WE12B4 (4.3 g) was separated on silica gel CC eluted with isocratic $\mathrm{CHCl}_{3}-\mathrm{MeOH}$ $(30: 70, v / v)$ to obtain WE12B41 and WE12B42. WE12B42 (1.4 g) was purified over silica gel CC eluted with $\mathrm{CHCl}_{3}$-EtOAc (4:1-2:1, v/v) and then further separated on semipreparative HPLC eluted with $\mathrm{MeCN}-\mathrm{H}_{2} \mathrm{O}(55: 45, v / v)$ to afford $1(15.1 \mathrm{mg})$. WE12B5 $(8.5 \mathrm{~g})$ was subjected to ODS CC eluted with $\mathrm{MeOH}-\mathrm{H}_{2} \mathrm{O}(55: 50-100: 0, v / v)$ to obtain four fractions (WE12B51-WE12B54). WE12B51 (4.0 g) was purified over silica gel CC eluted with petroleum ether-EtOAc (4:1-2:1, v/v) to yield 3 (100.8 mg). WE12B52 (2.3 g) was subjected to Sephadex LH-20 CC eluted with MeOH and then further purified over silica gel CC eluted with petroleum ether-EtOAc $(2: 1-1: 1, v / v)$ to yield 2 (20.3 mg).

WE13 (93 g) was separated over silica gel CC eluted with $\mathrm{CHCl}_{3}$-acetone $(10: 1-0: 1, v / v)$ to afford three fractions (WE13A-WE13C). WE13A (30.0 g) was subjected to MCI gel CC to yield three fractions (WE13A1-WE13A3). WE13A1 $(6.1 \mathrm{~g})$ was fractionated on silica gel CC eluted with $\mathrm{CHCl}_{3}$-acetone (10:1-8:1, v/v) to obtained three fractions (WE13A11-WE13A13). WE13A12 (680.2 mg) was subjected to Sephadex LH-20 CC eluted with $\mathrm{MeOH}$ and then further purified by semipreparative HPLC using MeCN-H $\mathrm{H}_{2} \mathrm{O}(40: 60, v / v)$ as mobile phase to yield 8 (201.1 mg). WE13A2 (4.1 g) was chromatographed on silica gel CC eluted with $\mathrm{CHCl}_{3}$-acetone $(10: 1-8: 1, v / v)$ to obtained three fractions (WE13A21-WE13A23). WE13A21 (401.3 mg) was separated on Sephadex LH-20 CC eluted with $\mathrm{CHCl}_{3}-\mathrm{MeOH}(1: 1, v / v)$ and then further purified over semipreparative HPLC eluted with $\mathrm{MeCN}-\mathrm{H}_{2} \mathrm{O}(49: 51, v / v)$ to yield $4(7.1 \mathrm{mg})$. WE13A23 was further purified over semipreparative HPLC eluted with MeCN-H ${ }_{2} \mathrm{O}(40: 60, v / v)$ to afford 6 (250.4 mg). WE13B was separated on ODS C18 CC eluted with $\mathrm{MeOH}-\mathrm{H}_{2} \mathrm{O}(50: 50-80: 20, v / v)$ to afford four fractions (WE13B1-WE12B4). WE13B2 was subjected to silica gel CC eluted with petroleum ether-EtOAc $(1: 2-1: 4, v / v)$ to yield WE13B21-WE13B23, all of which were purified over Sephadex LH-20 gel CC and then semipreparative HPLC. Consequently, compounds 9 (2.1 g), 11 (231.1 mg), and 5 (1.2 g) were isolated from WE13B21, WE13B22, and WE13B23, respectively. WE13B3 was also purified over Sephadex LH-20 gel CC and then semipreparative HPLC to afford 12 (150.4 mg).

WE2 (390.0 g) was separated on silica gel CC eluted with $\mathrm{CHCl}_{3}$-acetone $(6: 1-0: 1, v / v)$ to obtain WE21 and WE22. WE21 was firstly subjected to ODS CC eluted with $\mathrm{MeOH}-\mathrm{H}_{2} \mathrm{O}(40: 60-70: 20, v / v)$ to obtain WE21A-WE21D. WE21B was separated over silica gel CC eluted with $\mathrm{CHCl}_{3}$-acetone (6:1-0:1, $v / v$ ) to obtain four fractions (WE21B1-WE21B4). WE21B3 was chromatographed on ODS CC eluted with $\mathrm{MeOH}-\mathrm{H}_{2} \mathrm{O}(50: 50, v / v)$ to afford WE21B31 and WE21B32. Compounds 10 (420.7 mg) and 13 (420.4 mg) were isolated from WE21B31 and WE21B32, respectively, by semipreparative HPLC. WE21D was separated on silica gel CC eluted with $\mathrm{CHCl}_{3}$-acetone $(6: 1-0: 1, v / v)$ to obtain WE21D1 
and WE21D2. WE21D1 was purified over Sephadex LH-20 gel CC and then semipreparative HPLC to yield $14(100.8 \mathrm{mg})$ and $7(12.1 \mathrm{mg})$.

\subsection{1. (17S,23S)-17,23-Epoxy-3 $\beta, 15 \alpha$-dihydroxy-11-oxo-5 $\alpha$-lanosta-8-en-26,23-olide (1)}

White powder; $[\alpha] 20 D+75.7(c 0.12, \mathrm{MeOH})$; UV (MeOH) $\lambda_{\max }(\log \varepsilon) 258(3.84) \mathrm{nm}$; IR (KBr) $v_{\max } 3471,2975,2933,2869,1767,1635,1578,1454,1413,1378,1326,1287,1226,1186,1151,1119,1083$, 1042, 1006, 957, 921, $881 \mathrm{~cm}^{-1}$; For ${ }^{1} \mathrm{H}$ NMR and ${ }^{13} \mathrm{C}$ NMR spectroscopic data see Table 1; HRESIMS $\mathrm{m} / \mathrm{z} 499.3033[\mathrm{M}-\mathrm{H}]^{-}$(calcd. for $\mathrm{C}_{30} \mathrm{H}_{43} \mathrm{O}_{6}, 499.3065$ ).

\subsection{2. (17S,23S)-17,23-Epoxy-3 $\beta, 15 \alpha$-dihydroxy-7,11-dioxo- $5 \alpha$-lanosta-8-en-26,23-olide (2)}

White powder; $[\alpha] 20 D+85.2(c 0.13, \mathrm{MeOH}) ; \mathrm{UV}(\mathrm{MeOH}) \lambda_{\max }(\log \varepsilon) 202$ (3.55), 272 (3.85) nm; IR (KBr) $v_{\max } 3442,2967,2932,2866,1767,1665,1459,1384,1344,1263,1182,1158,1119,1095,1057,1027$, 961, 922, $882 \mathrm{~cm}^{-1}$; For ${ }^{1} \mathrm{H}$ NMR and ${ }^{13} \mathrm{C}$ NMR spectroscopic data, see Table 1; HRESIMS m/z 513.2852 $[\mathrm{M}-\mathrm{H}]^{-}$(calcd. for $\mathrm{C}_{30} \mathrm{H}_{41} \mathrm{O}_{7}, 513.2852$ ).

\subsection{3. (17S,23S)-17,23-Epoxy-3 $\beta, 7 \beta$-dihydroxy-11,15-dioxo-5 $\alpha$-lanosta-8-en-26,23-olide (3)}

White powder; $[\alpha] 20 D+104.1(c 0.12, \mathrm{MeOH})$; UV (MeOH) $\lambda_{\max }(\log \varepsilon) 201$ (3.79), 255 (4.17) nm; IR (KBr) $v_{\max } 3474,2974,2933,2870,1769,1729,1656,1455,1381,1329,1301,1265,1185,1139,1114$, 1073, 1037, 959, 916, $882 \mathrm{~cm}^{-1}$; For ${ }^{1} \mathrm{H}$ NMR and ${ }^{13} \mathrm{C}$ NMR spectroscopic data, see Table 1; HRESIMS $m / z 513.2855[\mathrm{M}-\mathrm{H}]^{-}$(calcd. for $\mathrm{C}_{30} \mathrm{H}_{41} \mathrm{O}_{7}, 513.2852$ ).

\subsection{4. (17S,23S)-17,23-Epoxy-3 $\beta, 7 \beta, 15 \alpha$-trihydroxy-11-oxo-5 $\alpha$-lanosta-8-en-26,23-olide (4)}

White powder; $[\alpha] 20 D+102.8$ (c 0.11, MeOH); UV (MeOH) $\lambda_{\max }(\log \varepsilon) 265$ (3.79) nm; IR (KBr) $v_{\max } 3490,3040,2976,2932,2867,1774,1750,1672,1455,1382,1328,1258,1188,1141,1075,1031,960$, 919, $883 \mathrm{~cm}^{-1}$; For ${ }^{1} \mathrm{H}$ NMR and ${ }^{13} \mathrm{C}$ NMR spectroscopic data, see Table 1; HRESIMS $\mathrm{m} / z$ 511.2700 $[\mathrm{M}-\mathrm{H}]^{-1}$ (calcd. for $\mathrm{C}_{30} \mathrm{H}_{39} \mathrm{O}_{7}$, 511.2696).

\subsubsection{3 $\beta$-Hydroxy-7,11,15,23-tetraoxo-5 $\alpha$-lanosta-8,16-dien-26-oic acid (5)}

White powder; $[\alpha] 20 D+111.0(c 0.23, \mathrm{MeOH}) ; \mathrm{UV}(\mathrm{MeOH}) \lambda_{\max }(\log \varepsilon) 234$ (4.02), 271 (3.80) nm; IR (KBr) $v_{\max } 3432,2968,2933,2872,1718,1677,1601,1459,1381,1189 \mathrm{~cm}^{-1}$; For ${ }^{1} \mathrm{H}$ and ${ }^{13} \mathrm{C}$ NMR data, see Table 2; HRESIMS $m / z 511.2684[\mathrm{M}-\mathrm{H}]^{-}$(calcd. for $\mathrm{C}_{30} \mathrm{H}_{39} \mathrm{O}_{7}, 511.2696$ ), $1023.5451[2 \mathrm{M}-\mathrm{H}]^{-}$ (calcd. for $\mathrm{C}_{60} \mathrm{H}_{79} \mathrm{O}_{14}, 1023.5470$ ).

\subsection{6. $3 \beta, 15 \alpha$-Dihydroxy-7,11,23-trioxo-5 $\alpha$-lanosta-8,16-dien-26-oic acid (6)}

White powder; $[\alpha] 20 D+68.6\left(c\right.$ 0.23, MeOH); UV (MeOH) $\lambda_{\max }(\log \varepsilon) 234$ (4.05), 272 (3.82) nm; IR $(\mathrm{KBr}) v_{\max } 3424,2970,2938,2875,1709,1686,1664,1461,1406 \mathrm{~cm}^{-1}$; For ${ }^{1} \mathrm{H}$ and ${ }^{13} \mathrm{C}$ NMR data, see Table 2; HRESIMS m/z 513.2843 [M-H] ${ }^{-}$(calcd. for $\mathrm{C}_{30} \mathrm{H}_{41} \mathrm{O}_{7}, 513.2852$ ), 1027.5767 [2M-H] ${ }^{-}$(calcd. for $\mathrm{C}_{60} \mathrm{H}_{83} \mathrm{O}_{14}, 1027.5783$ ).

\subsection{7. $3 \beta, 15 \alpha$-Dihydroxy-11,23-dioxo-5 $\alpha$-lanosta-8,16-dien-26-oic acid (7)}

White powder; $[\alpha] 20 D+97.7\left(c\right.$ 0.18, MeOH); UV (MeOH) $\lambda_{\max }(\log \varepsilon) 203$ (3.77), 259 (3.77) nm; IR (KBr) $v_{\max } 3430,2963,2932,2878,1715,1653,1577,1460,1375 \mathrm{~cm}^{-1}$; For ${ }^{1} \mathrm{H}$ and ${ }^{13} \mathrm{C}$ NMR data, see Table 2; HRESIMS m/z $499.3048[\mathrm{M}-\mathrm{H}]^{-}$(calcd. for $\mathrm{C}_{30} \mathrm{H}_{43} \mathrm{O}_{6}, 499.3060$ ), 999.6182 [2M-H] ${ }^{-}$(calcd. for $\mathrm{C}_{60} \mathrm{H}_{87} \mathrm{O}_{12}$, 999.6191).

\subsubsection{Bioassay}

$\alpha$-Glucosidase inhibitory activity was examined by the method described by Li et al. [22]. Acarbose, a definite $\alpha$-glucosidase inhibitor, was used as the positive control drug. 


\section{Conclusions}

Phytochemical investigation of G. resinaceum led to the isolation of 14 triterpenoids, including seven new triterpenoids (1-7) and seven known analogues (8-14) from the fruiting bodies of G. resinaceum. Compounds 1-4 and $\mathbf{8}$ were determined to be triterpenoid lactones which possessed an oxaspirolactone moiety in the side chain, consisting of a five-membered ether ring, a five-membered lactone ring, and a characteristic C-23 spiro carbon. It is rare that such an oxaspirolactone moiety ring occurred in the lanostane-type triterpenoids. Based on the analysis of plausible biogenetic pathway, compounds 5-7 and 9-14 may be important intermediates in the biosynthesis of 1-4 and 8. Compounds $\mathbf{1}$ and $\mathbf{2}$ showed stronger $\alpha$-glucosidase inhibitory activity than the positive control drug acarbose, which are strong $\alpha$-glucosidase inhibitors with $\mathrm{IC}_{50}$ value of $0.75 \pm 0.018 \mathrm{mM}$ and $1.64 \pm 0.022 \mathrm{mM}$, respectively.

Supplementary Materials: The following are available online, Spectrum copies of Mass, NMR, and IR, Table S1: Inhibition rate of compounds 3, 5, 6, 9, and 11-13 at the concentration of $3 \mathrm{mM}$.

Author Contributions: X.-Q.C. performed the isolation and structure elucidation of the chemicals, activity assay, and the preparation of the manuscript. L.-G.L. participated in structural elucidation of compounds. L.-X.C. takes part in the analysis of ESI-MS. Y.-P.T. and D.-L.L. both participated in the analysis of all experimental data. S.-P.L. and J.Z. planned organized the whole research of this study. All authors approved the final version manuscript.

Funding: This research was funded by the National Natural Science Foundation of China (Nos. 81673389 and 81603069), the Science and Technology Development Fund of Macau (No. 040/2016/A), and the Research Fund of University of Macau (No. MYRG2015-00122).

Conflicts of Interest: The authors declare no conflict of interest.

\section{References}

1. Oyetayo, O.V. Medicinal Uses of Mushrooms in Nigeria: Towards Full and Sustainable Exploitation. Afr. J. Tradit. Complement. Altern. Med. 2011, 8, 267-274. [CrossRef] [PubMed]

2. Peng, X.-R.; Liu, J.-Q.; Han, Z.-H.; Yuan, X.-X.; Luo, H.-R.; Qiu, M.-H. Protective effects of triterpenoids from Ganoderma resinaceum on $\mathrm{H}_{2} \mathrm{O}_{2}$-induced toxicity in HepG2 cells. Food Chem. 2013, 141, 920-926. [CrossRef] [PubMed]

3. Chen, X.-Q.; Zhao, J.; Chen, L.-X.; Wang, S.-F.; Wang, Y.; Li, S.-P. Lanostane triterpenes from the mushroom Ganoderma resinaceum and their inhibitory activities against $\alpha$-glucosidase. Phytochemistry 2018, 149, 103-115. [CrossRef] [PubMed]

4. Chen, X.Q.; Chen, L.X.; Zhao, J.; Tang, P.; Li, S.P. Nortriterpenoids from the Fruiting Bodies of the Mushroom Ganoderma resinaceum. Molecules 2017, 22, 1073. [CrossRef] [PubMed]

5. Chen, X.Q.; Chen, L.X.; Li, S.P.; Zhao, J. Meroterpenoids from the fruiting bodies of higher fungus Ganoderma resinaceum. Phytochem. Lett. 2017, 22, 214-218. [CrossRef]

6. Chen, X.Q.; Chen, L.X.; Li, S.P.; Zhao, J. A new nortriterpenoid and an ergostane-type steroid from the fruiting bodies of the fungus Ganoderma resinaceum. J. Asian Nat. Prod. Res. 2017, 19, 1239-1244. [CrossRef] [PubMed]

7. Zengin, G.; Sarikurkcu, C.; Gunes, E.; Uysal, A.; Ceylan, R.; Uysal, S.; Gungor, H.; Aktumsek, A. Two Ganoderma species: Profiling of phenolic compounds by HPLC-DAD, antioxidant, antimicrobial and inhibitory activities on key enzymes linked to diabetes mellitus, Alzheimer's disease and skin disorders. Food Funct. 2015, 6, 2794-2802. [CrossRef] [PubMed]

8. Amaral, A.E.; Carbonero, E.R.; Simao, R.D.C.G.; Kadowaki, M.K.; Sassaki, G.L.; Osaku, C.A.; Gorin, P.A.J.; Iacomini, M. An unusual water-soluble beta-glucan from the basidiocarp of the fungus Ganoderma resinaceum. Carbohydr. Polym. 2008, 72, 473-478. [CrossRef]

9. Niu, X.-M.; Li, S.-H.; Xiao, W.-L.; Sun, H.-D.; Che, C.-T. Two new lanostanoids from Ganoderma resinaceum. J. Asian Nat. Prod. Res. 2007, 9, 659-664. [CrossRef] [PubMed]

10. Silva, A.M.; Miranda, A.; Fernandes, E.; Santos, S.; Fraga, I.; Santos, D.L.; Dias, A.A.; Bezerra, R.M. Endopolysaccharides from Ganoderma resinaceum, Phlebia rufa, and Trametes versicolor Affect Differently the Proliferation Rate of HepG2 Cells. Appl. Biochem. Biotechnol. 2013, 169, 1919-1926. [CrossRef] [PubMed] 
11. Ofodile, L.N.; Uma, N.U.; Kokubun, T.; Grayer, R.J.; Ogundipe, O.T.; Simmonds, M.S.J. Antimicrobial activity of some Ganoderma species from Nigeria. Phytother. Res. 2005, 19, 310-313. [CrossRef] [PubMed]

12. Li, Y.-L.; Gao, Y.-X.; Jin, H.-Z.; Shan, L.; Chang, W.-L.; Yang, X.-W.; Zeng, H.-W.; Wang, N.; Steinmetz, A.; Zhang, W.-D. Chemical constituents of Abies fabri. Phytochemistry 2015, 117, 135-143. [CrossRef] [PubMed]

13. Wang, G.-W.; Lv, C.; Fang, X.; Tian, X.-H.; Ye, J.; Li, H.-L.; Shan, L.; Shen, Y.-H.; Zhang, W.-D. Eight Pairs of Epimeric Triterpenoids Involving a Characteristic Spiro-E/F Ring from Abies faxoniana. J. Nat. Prod. 2015, 78, 50-60. [CrossRef] [PubMed]

14. Liu, L.-Y.; Chen, H.; Liu, C.; Wang, H.-Q.; Kang, J.; Li, Y.; Chen, R.-Y. Triterpenoids of Ganoderma theaecolum and their hepatoprotective activities. Fitoterapia 2014, 98, 254-259. [CrossRef] [PubMed]

15. Zhang, S.-S.; Wang, Y.-G.; Ma, Q.-Y.; Huang, S.-Z.; Hu, L.-L.; Dai, H.-F.; Yu, Z.-F.; Zhao, Y.-X. Three New Lanostanoids from the Mushroom Ganoderma tropicum. Molecules 2015, 20, 3281-3289. [CrossRef] [PubMed]

16. Guan, S.-H.; Yang, M.; Wang, X.-M.; Xia, J.-M.; Zhang, Z.-M.; Liu, X.; Guo, D.-A. Spectral assignments and reference data structure elucidation and complete NMR spectral assignments of three new lanostanoid triterpenes with unprecedented $\Delta^{16,17}$ double bond from Ganoderma lucidum. Magn. Reson. Chem. 2007, 45, 789-791. [CrossRef] [PubMed]

17. Hu, L.-L.; Ma, Q.-Y.; Huang, S.-Z.; Guo, Z.-K.; Ma, H.-X.; Guo, J.-C.; Dai, H.-F.; Zhao, Y.-X. Three new lanostanoid triterpenes from the fruiting bodies of Ganoderma tropicum. J. Asian Nat. Prod. Res. 2013, 15, 357-362. [CrossRef] [PubMed]

18. Lin, C.N.; Kuo, S.H.; Won, S.J. Steroids of Formosan Ganoderma amboinense. Phytochemistry 1993, 32, $1549-1551$.

19. Kikuchi, T.; Kanomi, S.; Kadota, S.; Murai, Y.; Tsubono, K.; Ogita, Z.I. Constituents of the fungus Ganoderma lucidum (Fr.) Karst. I. Structures of ganoderic acids C2, E, I, and K, lucidenic acid F and related compounds. Chem. Pharm. Bull. 1986, 34, 3695-3712. [CrossRef]

20. Kikuchi, T.; Kanomi, S.; Murai, Y.; Kadota, S.; Tsubono, K.; Ogita, Z. Constituents of the fungus Ganoderma lucidum (Fr.) Karst. III. Structures of ganolucidic acids A and B, new lanostane-type triterpenoids. Chem. Pharm. Bull. 1986, 34, 4030-4036. [CrossRef]

21. Hasegawa, S.; Kaneko, N.; Hirose, Y. Triterpenes from the seed of Abies firma. Phytochemistry 1987, 26, 1095-1099. [CrossRef]

22. Li, D.-Q.; Qian, Z.-M.; Li, S.-P. Inhibition of Three Selected Beverage Extracts on $\alpha$-Glucosidase and Rapid Identification of Their Active Compounds Using HPLC-DAD-MS/MS and Biochemical Detection. J. Agric. Food. Chem. 2010, 58, 6608-6613. [CrossRef] [PubMed]

Sample Availability: Samples of the compounds 1-14 are available from the corresponding authors.

(C) 2018 by the authors. Licensee MDPI, Basel, Switzerland. This article is an open access article distributed under the terms and conditions of the Creative Commons Attribution (CC BY) license (http:/ / creativecommons.org/licenses/by/4.0/). 